\title{
Desarrollo de proyectos de investigación científica en el contexto Norte-Sur
}

Iving Zelaya Perdomo ${ }^{1}$

La poca relevancia que se otorga a la función de investigación en la sociedad y en las propias universidades del Sur, la debilidad de los sistemas nacionales de ciencia y tecnología y la falta de capacidades disciplinares y metodológicas aunada a las carencias en equipamiento e infraestructura cualificada para la investigación en las instituciones de educación superior son elementos que inciden sobre el mantenimiento de la brecha norte-sur en investigación científica.

En este artículo se da cuenta de algunos elementos concretos que, en el caso de Honduras y la UNAH, propician esta brecha u obstaculizan su reducción; al tiempo que se describen algunas particularidades concernientes al desarrollo de proyectos de investigación colaborativos en el contexto Norte-Sur y se realizan apuntes que podrían aportar a la construcción de políticas públicas que potencien la producción científica como factor clave del desarrollo y faciliten la labor de los gestores académicos de investigación en el nivel de educación superior.

\section{LA BRECHA NORTE-SUR EN INVESTIGACIÓN CIENTÍFICA}

Uno de los elementos que influye significativamente en la brecha que existe entre el volumen de la producción científica y su incidencia en países del Norte y el Sur global es la relevancia que se otorga a la práctica de la investigación académica y, consecuentemente, el contexto y condiciones en las que los investigadores desarrollan sus proyectos.

Es posible aseverar que, en el caso de Honduras y otros países de la región, la educación superior, la actividad científica y la innovación no se encuentran entre los temas prioritarios de las agendas de trabajo nacionales. El conjunto social no considera la investigación y la producción científica como elementos torales para los procesos de desarrollo, y la construcción de políticas públicas ligada a ejercicios rigurosos de generación de conocimiento es muy escasa.

${ }^{1}$ Profesora de la Dirección de Investigación Científica de la Universidad Nacional Autónoma de Honduras: investigacionunah.gestion.iz@gmail.com 
En el año 2017, Honduras ocupaba el puesto 96 de un total de 137 economías consideradas en el Índice de Competitividad Global, instrumento que mide el conjunto de instituciones, políticas y factores que inciden sobre la productividad del país y su influencia sobre la prosperidad económica sostenible y la tasa de retorno de las inversiones que se realizan en territorio nacional; un retroceso de ocho posiciones con respecto al año anterior.

Entre los 12 pilares que estructuran este índice, se encuentran la Educación Superior y Formación, como categoría "crucial para escalar en las cadenas de valor más allá de los meros procesos productivos y productos" (Schwab \& Sala-i-Martín, 2017); y la innovación. En el primero, que incluye entre sus componentes las tasas brutas de matrícula en educación secundaria y terciaria, la educación continua o la calidad de la educación de acuerdo a la percepción de líderes empresariales, Honduras ocupa la posición 102 de 137. En el pilar de innovación, focalizado en la existencia y mantenimiento de un ambiente que propicie la actividad de innovación impulsada desde los ámbitos público y privado, el país ocupa la posición 108 de 137.

\section{Índice de competitividad global 2017-2018 para Honduras pilares 5: educación superior y formación y 7: innovación}

\begin{tabular}{|l|l|l|l|}
\hline & & Ranking /137 & Valor \\
\hline Pilar $\mathbf{5}$ & Educación Superior y Formación & 102 & ${ }^{*} 3.6$ \\
\hline 5.01 & Tasa bruta de matrícula en educación secundaria & 98 & 70.8 \\
\hline 5.02 & Tasa bruta de matrícula en educación terciaria & 93 & 22.1 \\
\hline 5.03 & Calidad del sistema educativo & 98 & ${ }^{*} 3.2$ \\
\hline 5.04 & Calidad de la educación matemática y científica & 114 & ${ }^{*} 3.1$ \\
\hline 5.05 & Calidad de las escuelas de administración & 110 & ${ }^{*} 3.6$ \\
\hline 5.06 & Acceso a Internet en las escuelas & 102 & ${ }^{*} 3.6$ \\
\hline 5.07 & Disponibilidad local de servicios de capacitación especializados & 106 & ${ }^{*} 3.8$ \\
\hline 5.08 & Alcance de la formación del personal & 59 & ${ }^{*} 4.1$ \\
\hline & & Ranking /137 & Valor \\
\hline Pilar $\mathbf{1 2}$ & Innovación & 108 & ${ }^{*} 2.9$ \\
\hline 12.01 & Capacidad para la Innovación & 86 & ${ }^{*} 3.9$ \\
\hline 12.02 & Calidad de las instituciones de investigación científica & 120 & ${ }^{*} 2.8$ \\
\hline 12.03 & Gastos de empresas en I + D & 102 & ${ }^{*} 2.9$ \\
\hline 12.04 & Colaboración universidad-industria en I + D & 101 & ${ }^{*} 3.0$ \\
\hline 12.05 & Contratación del gobierno de productos de tecnología avanzada & 112 & ${ }^{*} 2.8$ \\
\hline 12.06 & Disponibilidad de científicos e ingenieros & 96 & ${ }^{*} 3.6$ \\
\hline 12.07 & Solicitudes de patentes PCT / millón de personas & 119 & ${ }^{*} 0.0$ \\
\hline${ }^{*}$ Escala del 1-7 & & \\
\hline
\end{tabular}

Fuente: The Global Competitiveness Report. 2017-2018 
La histórica debilidad del sistema nacional de ciencia y tecnología en Honduras podría considerarse una de las causas de la baja productividad reflejada en la medición de estas categorías. "En 1993, el gobierno hondureño crea el Consejo Hondureño de Ciencia y Tecnología (COHCIT), con el propósito de promover, estimular, y fortalecer el desarrollo científico y tecnológico en Honduras. En esta etapa el COHCIT elaboró un Programa de Desarrollo Científico y Tecnológico (...) que no fue aprobado. En el 2010, las funciones del COHCIT se absorbieron por la Dirección Nacional de Competitividad e Innovación de la Secretaria Técnica de Planificación y Cooperación (SEPLAN), pero no se priorizó la competitividad tecnológica." (Instituto Hondureño de Ciencia, Tecnología y la Innovación, 2018).

En 2014, el Estado hondureño aprobó, mediante decreto No. 276-2013, la creación del "Sistema Nacional de Ciencia, Tecnología y la Innovación", que actualmente funciona de manera parcial a través del Instituto Hondureño de Ciencia, Tecnología y la Innovación (IHCIETI): una instancia orientada, según lo establecido en el plan de gobierno 2014-2018, a "implementar el Sistema Nacional de la Calidad, la Política Nacional de Metrología, la Agenda Nacional de Ciencia e Investigación y la Agenda Digital de Honduras". (Instituto Hondureño de Ciencia, Tecnología y la Innovación, 2018)

En un contexto en el que los mecanismos de financiamiento e incentivos para el desarrollo de la investigación a través del citado sistema nacional de ciencia, tecnología y la innovación son escasos, no necesariamente vinculados a las instituciones de educación superior y focalizados en la investigación aplicada para el desarrollo tecnológico de cinco áreas priorizadas, las universidades públicas de Honduras tienen una enorme responsabilidad en el impulso de ejercicios de investigación vinculados a la construcción de políticas públicas que coadyuven al logro de todos y cada uno de los objetivos y lineamientos instituidos en la vigente Ley para el establecimiento de una visión de país y la adopción de un plan de nación para Honduras aprobada mediante decreto $286 / 2009$.

Sin embargo, tradicionalmente, las funciones universitarias de investigación y vinculación han sido consideradas como labores colaterales de los profesores universitarios en las distintas instituciones de educación superior públicas y privadas de Honduras. "La Universidad Nacional Autónoma de Honduras (UNAH), como todas las universidades del país y casi todas las de la región centroamericana fue durante muchos años una universidad de docencia de grado que ha ido evolucionando hacia una universidad de grado, posgrado y de investigación, en un proceso lento que ha enfrentado numerosas dificultades originadas en el hecho de que los profesores 
universitarios ingresan cumpliendo funciones exclusivamente de docencia de grado, minimizando o marginando las otras dos funciones, en particular la de investigación." (Dirección de Investigación Científica y Posgrado, UNAH, 2018).

Cabría considerar la posibilidad de que el protagonismo de la función de docencia, en detrimento del desarrollo de la investigación científica básica y aplicada en la universidad, guardara relación con los bajos niveles de formación de posgrado del cuerpo de profesores de la institución. Según datos oficiales, en el año 2013 solamente un $3 \%$ de los profesores de la UNAH contaba con el grado académico de doctorado, mientras que el $33 \%$ contaba con el grado de especialidad o maestría. Esta cifra se considera consistente con los datos presentados por la UNESCO (2015) que cuantifican anualmente dos (2) graduados de doctorado por cada millón de habitantes en Honduras.

La UNAH contaba en el año 2015 con una plantilla compuesta por 3,773 profesores (Banco Central de Honduras, 2018) de los que solamente 103 llenaron los requisitos para figurar en el Catálogo de Investigadores y sus Publicaciones de la UNAH (Dirección de Investigación Científica y Posgrado, UNAH, 2016). Superar las carencias en cuanto a formación especializada del profesorado en los ámbitos disciplinar y tecnológico se complejiza debido a factores como la poca inversión en programas de movilidad y la persistencia de procesos administrativos burocráticos que obstaculizan la gestión de iniciativas académicas de fomento a la actividad científica.

Las condiciones de equipamiento e infraestructura para el desarrollo de la investigación son también determinantes en la UNAH. Aunque los investigadores consiguen acceder cada vez más a tecnologías recientes, tanto la efectividad en la cualificación de áreas de trabajo y equipos como la falta de prácticas adecuadas para su mantenimiento y el difícil acceso a insumos necesarios para el ejercicio académico siguen siendo una limitante.

A diferencia de lo que generalmente sucede en los países del Norte, estas condiciones son comunes en muchas universidades públicas de la región centroamericana; en contraste, en el Norte global la investigación es considerada fundamental y su importancia sobrepasa en muchas ocasiones a la otorgada a la función de docencia debido a la trascendencia del impacto en sus resultados en las agendas de desarrollo nacionales y regionales.

Las cualificaciones de acceso a las instituciones de educación superior son más rigurosas, propiciando que las plantillas de profesores de las universidades ostenten 
los mayores grados académicos y una trayectoria en investigación reflejada a través de numerosas científicas. No solamente se cuenta con más y mejores infraestructuras físicas y equipamiento; también es posible acceder a diversos mecanismos de obtención de recursos para el desarrollo de procesos de investigación, desarrollo tecnológico e innovación a través de organismos nacionales y supranacionales. Las instituciones de educación superior en el Norte global desarrollan su actividad en un paradigma distinto, en el que los profesores pueden focalizar la investigación como tarea prioritaria en su trabajo académico.

\section{DESARROLLO DE PROYECTOS DE INVESTIGACIÓN COLABORATIVOS}

"La evolución de la ciencia y la tecnología está caracterizada, en los países industrializados del Norte, por la creciente especialización de la investigación y el desarrollo, la acelerada diversificación de los conocimientos teóricos y prácticos y la progresiva descentralización de los servicios de investigación. Existe el peligro de que la dinámica de esta evolución aumente todavía más la diferencia entre el Norte y el Sur en materia de investigación, que ya es muy grande. Por esta razón se ha concedido alta prioridad en los órdenes del día internacionales al fortalecimiento de los servicios de investigación en el Sur, a la utilización en común de recursos mediante distintos sistemas de cooperación Norte-Sur y Sur-Sur y a la mejora del acceso en todo el mundo a la información sobre investigación científica disponible en el Norte." (UNCTAD, 1999)

Es posible aducir que los investigadores en Honduras se han mostrado anuentes al desarrollo de proyectos colaborativos con pares localizados en países del Norte. De acuerdo con los datos de la Organización de las Naciones Unidas para la educación, la ciencia y la cultura (UNESCO, 2015) un $97,6 \%$ de los artículos cientíicos publicados por investigadores hondureños en revistas indexadas tienen una coautoría extranjera, encontrándose la mayor parte de socios académicos en Estados Unidos. Este dato es coherente con la información disponible en el Instituto de Estadística de la UNESCO (UIS), que reflejan que Estados Unidos es el principal destino de movilidad escogido por los estudiantes hondureños que realizar estudios superiores en el extranjero; quienes posteriormente funcionan como motores para el establecimiento de relaciones de cooperación académica con universidades y centros de investigación internacionales.

Sin embargo, es necesario precisar que la participación de los investigadores en las 
redes de colaboración Norte-Sur para la investigación no es siempre equilibrada, "ya que los asociados del Norte, que suelen seguir dominando en dichas redes, tienden a reducir la importancia del desarrollo. Normalmente disponen de mayores fondos que sus colegas del Sur para desarrollar las ideas contenidas en los proyectos en colaboración. Frecuentemente «bombean» el grueso de la información por la red con la consecuente ganancia tanto de perspectiva como de influencia en el establecimiento de programas de la red. Los desequilibrios existentes en muchas redes de investigación Norte-Sur reducen su importancia para el desarrollo." (UNCTAD, 1999) Adicionalmente, los mecanismos utilizados para la medición de la capacidad científica propugnados por el Norte tienen su fundamento en indicadores bibliométricos "utilizados para medir la actividad científica que se basan en los recuentos de las publicaciones y de las citas recibidas por los trabajos publicados, así como en el impacto de las revistas de publicación" (Bordons \& Zulueta, 1999). El problema para los investigadores del Sur reside en que las bases de datos utilizadas en estas mediciones "no reflejan la producción de conocimientos a escala internacional, sino una porción de esas investigaciones, las que se publican en inglés, bajo las normas de un dispositivo de jerarquización del conocimiento conducido por empresas editoriales y dominado por algunos «centros de excelencia» (...). La "citometría" no es un instrumento neutro de medición de prestigio científico universal, sino la herramienta principal de una estructura internacional de recursos y capacidades de investigación crecientemente desigual, que se manifiesta en el volumen de la producción científica, en los flujos migratorios de población calificada, en la universalización de estándares de publicación y en la abrumadora supremacía del inglés como lengua franca internacional". (Beigel, 2014)

Reducir la asimetría de las relaciones entre investigadores del Norte y del Sur se antoja como una tarea complicada en tanto sean los socios de los países industrializados quienes lideren las iniciativas para el desarrollo de proyectos, que generalmente estarán insertos en sus propias agendas de investigación, y para el establecimiento de redes científicas internacionales en las que los investigadores del Sur son meros invitados.

De acuerdo a la UNCTAD (1999), la participación real de los socios del Sur en la planificación, gestión y desarrollo de proyectos de investigación y en el establecimiento de redes se ha convertido en una condición de peso en la valoración de propuestas por los entes financiadores. El conocimiento de los contextos locales y las posibilidades de acceso a la información son también elementos que potencian el papel de los investigadores del Sur en las colaboraciones académicas, cambiando su rol al de participantes activos en los procesos emprendidos de manera conjunta. 
A la vez, se ha abierto la posibilidad para la gestión de recursos a través de los mecanismos de ayuda oficial al desarrollo con el fin de "promover el progreso tecnológico, científico e innovador de los países en desarrollo" (Quiñones Montellano \& Tezanos Vázquez, 2011) y disminuir la brecha existente a través de proyectos que involucran a investigadores, instituciones y otros actores de ambos hemisferios.

Características de las políticas internacionales de cooperación científicotecnológicas para el desarrollo

\section{Objetivos:}

1. Crear sistemas nacionales de investigación sólidos

2. Transferir conocimiento y tecnología

3. Formar y capacitar de recursos humanos

4. Facilitar la movilidad de investigadores

5. Facilitar el aprendizaje tecnológico

6. Crear infraestructuras de I+D+i

7. Sensibilizar al conjunto de la sociedad sobre la relevancia de la ciencia, la tecnología y la innovación

8. Satisfacer las demandas nacionales de innovación

9. Recuperar conocimientos tecnológicos locales

\section{Modalidades de ayudas:}

1. Ayudas para la investigación y el desarrollo tecnológico (agrícola, forestal, pesquera, educativa, sanitaria, energética y medioambiental).

2. Ayudas para la adquisición de competencias avanzadas y específicas (formación profesional, enseñanza superior, fomento de capacidades estadísticas, extensión agraria y varios tipos de enseñanza y formación referida a sectores sociales, productivos y comerciales).

Poco a poco, la tendencia al desarrollo de proyectos con socios de Estados Unidos y Europa Occidental ha empezado a modificarse, reflejándose mayores niveles de colaboración para la publicación científica conjunta con investigadores pertenecien- 
tes a otros países del Sur; de acuerdo con la UNESCO (2015), en el caso de Honduras se evidencian considerables interacciones con investigadores de México, Brasil, Argentina y Colombia, por ese orden.

Según lo planteado por la UNCTAD (1999), las redes regionales de investigación Sur-Sur han tenido un desempeño más eficiente producto del desarrollo de colaboraciones más horizontales en las que los intereses científicos y las prioridades de investigación son comunes y están relacionadas con los propios contextos económicos, sociales y culturales. Estos intercambios han probado ser más exitosos en experiencias con altos niveles de movilidad internacional en las que los investigadores participan en todas las fases del desarrollo de los proyectos a través de mecanismos que establecen oficialmente su dedicación a las actividades programadas y en las que los productos se encuentran claramente establecidos.

\section{CONSIDERACIONES FINALES}

Las universidades públicas del Sur son actores determinantes para la disminución de la brecha científico-tecnológica que existe en la actividad de investigación con respecto a los países del Norte. La tarea de potenciar la participación de los investigadores de Honduras y Centroamérica en iniciativas de cooperación académica en las que no funjan como meros beneficiarios de acciones diseñadas en el Norte, sino más bien como parte activa de una construcción colectiva derivada de un diálogo entre pares, puede impulsarse desde las instituciones de educación superior.

El primer imperativo es la construcción de agendas de investigación propias que contribuyan a la solución de problemas nacionales y regionales, que brinden elementos para la elaboración de políticas públicas en los muy diversos aspectos que ocupan al gobierno y a los ciudadanos, y que potencien la innovación a la vez que aportan al desarrollo de la ciencia y a la producción de un conocimiento inclusivo que refleje las condiciones sociopolíticas y culturales del ámbito local.

Para que estas agendas puedan desarrollarse de manera efectiva, las universidades deben incidir sobre la creación de sistemas nacionales de investigación fuertes e incluyentes. Estos servirán para impulsar el fortalecimiento de capacidades de investigación y potenciar los liderazgos científicos del país, enfatizando el desarrollo de posgrados de calidad y la mejora de las condiciones de infraestructura científica y tecnológica; para potenciar el desarrollo de proyectos de investigación contextualiza- 
dos y propiciar una divulgación pertinente de los conocimientos científicos y tecnológicos generados; para reducir los obstáculos burocráticos en el desarrollo de la actividad científica y construir estándares de calidad propios y adecuados para la medición de la actividad científica en el Sur; y para orientar los esfuerzos para la gestión de la cooperación académica y fomentar la conformación de grupos de investigación temáticos compuestos mayoritariamente por socios del Sur, que de acuerdo a lo propugnado por Gibbons (1998) sean capaces de interactuar con otros productores de conocimiento para aprovechar los saberes de manera creativa y realizar investigación para la generación de conocimiento en el marco de su contexto de aplicación.

Revertir la hegemonía científica tradicional y vincular la investigación a la construcción y puesta en marcha de políticas públicas para el desarrollo de país requiere que la institucionalidad, los elaboradores de políticas y tomadores de decisiones, y la sociedad otorguen la debida relevancia al desarrollo de la educación superior y específicamente a la función de investigación. La articulación de los intereses científicos con los contextos locales es indispensable para fortalecer la legitimidad de las universidades públicas.

\section{REFERENCIAS BIBLIOGRÁFICAS}

Banco Central de Honduras. (2018). Honduras en Cifras. 2015-2017. Tegucigalpa: BCH. Beigel, F. (2014). Centros y periferias en la circulación internacional del conocimiento. Nueva Sociedad(245), 110-123.

Bordons, M., \& Zulueta, M. Á. (1999). Evaluación de la actividad científica a través de indicadores bibliométricos. Revista Española de Cardiología, 52(10), 790-800.

Congreso Nacional de Honduras. (31 de Mayo de 2018). Decreto Legislativo No. 286-2009,

Ley para el Establecimiento de una Visión de País y la Adopción de un Plan de Nación para Honduras. . Obtenido de http://www.sre.gob.hn: http://www.sre.gob.hn

Dirección de Investigación Científica y Posgrado, UNAH. (2016). Catálogo de Investigadores y sus Publicaciones. UNAH-2015. Tegucigalpa: DICyP-UNAH.

Dirección de Investigación Científica y Posgrado, UNAH. (2018). 12 años de reforma en investigación científica y 5 años de reforma en posgrado. Tegucigalpa: DICyP-UNAH. Gibbons, M. (1998). Higher education relevance in the 21 rst century. Washington D.C.: World Bank.

Instituto Hondureño de Ciencia, Tecnología y la Innovación. (4 de junio de 2018). Plan Estratégico Institutional. Obtenido de https://www.senacit.gob.hn: https://www.senacit.gob.hn/documentos/PLAN-ESTRATEGICO-INSTITUCIONAL-IHCIETI-2018.pdf 
Quiñones Montellano, A., \& Tezanos Vázquez, S. (2011). Ayuda oficial al desarrollo científico-tecnológica: una evaluación macroeconómica de la distribución geográfica y sectorial. Revista de Economía Mundial(29), 153-179.

Schwab, K., \& Sala-i-Martín, X. (2017). The Global Competitiveness Report. 2017-2018. Ginebra: World Economic Forum.

UNCTAD. (1999). Funcionamiento de las redes de investigación norte-sur. Ginebra: UNCTAD.

UNESCO. (2015). UNESCO Science Report: towards 2030. Paris: UNESCO Publishing. 